\title{
Aroma of Eucommia leaf extract (ELE) causes reduced locomotor activity and increased NREM sleep, acting like the partially related factors of oral ELE's effects with locomotor-activity-dependent-increase in NREM- and REM-sleep
}

\author{
Shouhei Miyazaki ${ }^{1}$, Hirotaka Oikawa ${ }^{1}$, Shoko Nakamichi ${ }^{1}$, Tetsuya Hirata ${ }^{2}$, Hiroo Yamasaki ${ }^{2}$, Yasuyo Yamaguchi ${ }^{2}$, Wenping Zhang ${ }^{3}$, Sansei \\ Nishibe $^{4}$, Masato Ogata ${ }^{5}$ and Takahiko Fujikawa ${ }^{1,5 *}$ \\ ${ }^{1}$ Laboratory of Molecular Prophylaxis and Pharmacology, Faculty of Pharmaceutical Sciences, Suzuka University of Medical Science, 3500-3 Suzuka, Minamitamagaki- \\ cho, Susuka, Mie 513-8670, Japan \\ ${ }^{2} \mathrm{R} \& D$ Center, Kobayashi Pharmaceutical Co., Ltd. 1-30-3, Toyokawa, Ibaraki, Osaka 567-0057, Japan \\ ${ }^{3}$ Faculty of Acupuncture \& Moxibustion, Suzuka University of Medical Science, Mie, Japan \\ ${ }^{4}$ Faculty of Pharmaceutical Sciences, Health Sciences University of Hokkaido, 1757 Kanazawa, Tobetsu-cho, Ishikari, Hokkaido 061-0293, Japan \\ ${ }^{5}$ Department of Biochemistry and Proteomics, Mie University Graduate School of Medicine, 2-174 Edobashi, Tsu, Mie 514-8507, Japan
}

\begin{abstract}
The roasted leaves of Eucommia ulmoides are used for preparing tea in Japan. Eucommia leaf extract (ELE) has been known to have antihypertensive and anti-obesity effects. This study showed that an oral ELE administration or its aroma treatment had hypnotic effects, and these effects depended on a decrease in the locomotor activity with both treatments during the entire period, particularly in the light period. In addition, the reduction of the locomotor activity in the light period using an oral ELE administration was based on the induction of NREM and REM sleep. However, the reduction of the locomotor activity in the light period using an aroma treatment with ELE was due to the induction of NREM sleep. In other words, the mechanism of action of aromatic treatment of ELE is potentially similar to that of the oral administration with ELE. Moreover, the ELE aroma, which resulted in reduction of locomotor activity and induction of NREM sleep may act as the partially related factors of oral ELE's effects with locomotor-activity-dependent increase in the NREM and REM sleep in the light period. Thus, instead of using insomnia medicines that have side effects, various administration methods of ELE may be one of the best choices in treating insomnia. Ingestion or inhalation of ELE, which is a type of food, improves the sleeping pattern and prevents the progression of insomnia.
\end{abstract}

\section{Introduction}

Sleep is an important behavior that allows the body and brain to rest. Currently, insomnia is generally recognized as a public health problem, which results in insomnia-induced daytime fatigue, impaired daytime functioning, and decreased quality of life. Insomnia has been reported to be related to depression [1,2] and metabolic syndrome [3]. A survey conducted by the Japanese Ministry of Health, Labour and Welfare indicated that many Japanese people have various insomnia symptoms (i.e., difficulty falling asleep, prolonged nocturnal awakening, and undesired early morning awakening) [4]. Sleep is classified into rapid eye movement (REM) sleep and non-REM (NREM) sleep. Furthermore, NREM sleep is classified into four stages. REM sleep is associated with rapid eye movement and relaxation of the skeletal muscle. The electroencephalogram (EEG) of the REM sleep shows awake EEG (high frequency, low amplitude, and desynchronization) while being in a sleep state; hence, REM sleep is also called paradoxical sleep (PS). NREM is the phase defined as any sleep excluding REM sleep. The EEG of NREM sleep shows high amplitude and synchronization. REM and NREM sleep tend to alternate at 90 min interval in humans. In rats,
Correspondence to: T Fujikawa, Laboratory of Molecular Prophylaxis and Pharmacology, Faculty of Pharmaceutical Sciences, Suzuka University of Medical Science, Minamitamagaki-cho, Susuka, Mie 513-8670, Japan; E-mail: fujikawa@suzuka-u.ac.jp

Key words: Eucommia leaf extract (ELE), locomotor activity, insomnia, quality of life, rapid eye movement

Special Issue: Application to prophylactic pharmacology of food

Hirotaka Oikawa

Assistant professor

Department of Pharmaceutical Sciences

Suzuka University of Medical Science

Japan

Takahiko Fujikawa

Professor

Suzuka University of Medical Science Japan

Received: September 20, 2017; Accepted: October 20, 2017; Published: October 26, 2017 
Miyazaki S (2017) Aroma of Eucommia leaf extract (ELE) causes reduced locomotor activity and increased NREM sleep, acting like the partially related factors of oral ELE's effects with locomotor-activity-dependent-increase in NREM- and REM-sleep

the alternation occurs at 20 min interval; however, this sleep cycle is weak and variable. Sleep is associated with various neurotransmitter in humans and rats [5-7]. It is affected by various factors (i.e., age, sex, temperature, light, alcohol, medicine, stress, etc.). Sedative-hypnotics initiate, sustain, or lengthen the sleep. The following medicinal substrates are well known sedatives and hypnotics: barbiturates, benzodiazepines, nonbenzodiazepines, suvorexant (selective orexin receptor antagonist), and ramelteon (selective melatonin receptor agonist). Barbiturates and benzodiazepines among the sedativehypnotics inhibit REM sleep and slow-wave sleep. The reduced REM sleep induces a feeling of hangover in humans. The slow-wave sleep is related to quality of sleep; therefore, these medicines seem insufficient in treating insomnia in some people. However, aromatherapy and cognitive behavioral therapy also existed as treatment of insomnia without using medicines. A sleep induction effect caused by inhaling essential oils derived from many plants has been reported $[8,9]$. The inhalation of aroma with hypnotic properties may provide a safe and effective therapy for some people suffering from insomnia.

Eucommia ulmoides Oliver (named Duzhong or tuchung in China and Tochu in Japan) is the only known species of the genus Eucommia. The bark of E. ulmoides (Cortex Eucommia) has been traditionally used as an herbal medicine with analeptic, analgesic, sedative, antihypertensive, and diuretic effects in Japan, China, and Korea. In vitro and in vivo studies indicated that the compounds and extracts from Eucommia ulmoides have various pharmacological effects in treating hypertension [10-13], hyperlipidemia [14,15], diabetes [16], obesity [14, 17-20], osteoporosis [21-26], and lupus-like syndrome [27], and promoting neuroprotection [28,29] and immunoregulation [30]. E. ulmoides leaves and barks have the same iridoid and lignin compound components; therefore, the leaves have been used similarly as the bark, as tonic and antihypertensive. Because we can obtain the leaf through one year, we can always use it as a tea break for health maintenance. Administration of E. ulmoides leaf extract (ELE) and its ingredients showed dose-dependent antihypertensive effects in spontaneously hypertensive rats [31,32]. Antihypertensive effects of ELE are related to activation of the parasympathetic nervous system [31] and angiotensin-converting enzyme (ACE) inhibitory effect [33]. For this antihypertensive effect, the tea made from the roasted leaves of Eucommia ulmoides (Tochu-cha in Japanese) is used as a functional food. Some monomers from male flowers and leaves of E. ulmoides have been reported to have sedative and hypnotic effects [34,35].

Our laboratory data previously reported that asperuloside stimulates metabolic function across several organs in rats under highfat diet conditions, which act as the major ingredient in the anti-obesity and anti-metabolic syndrome activities of ELE [19,36]. In addition, ELE has been found to cause the suppression of appetite, which is partly involved in an anti-obesity effect [37]. The video-recording and locomotor activity measurement showed that the induction of sleep behavior using ELE caused a decreased chance (absolute time) of eating in rats, which accordingly lead to a decrease in visceral fats.

In this study, the effects of oral or aromatic treatment have been investigated using ELE on sleep behavior and quality based on the analysis of locomotor activity and EEG. Furthermore, we determined whether the ELE aroma contributes to the effects of oral ELE.

\section{Materials and methods}

\section{Plant extract}

The leaves of Eucommia collected in the Sichuan District of China were used. ELE was prepared as follows: Fresh Eucommia leaves were treated with steam at $100-110^{\circ} \mathrm{C}$ and then dried and roasted. Two tons of roasted Eucommia leaves were steeped in 10 tons of hot water at $90^{\circ} \mathrm{C}$ for $1 \mathrm{~h}$, and the extract was filtered and concentrated. The concentrate was left to stand for one day. Then, it was filtered and concentrated again, vacuum-dried, and powdered (yield: 18\%), as described [19].

\section{Animals}

Male Sprague-Dawley (SD) rats (7 weeks old) were purchased from the SLC, Inc. (Shizuoka, Japan). The rats were housed individually in standard polycarbonate cages for 7 days and subjected to serial 7-day handling; a normal diet (ND: MF, Oriental Yeast Manufacturing Co., Ltd., Tokyo, Japan) and water were available ad libitum. The rats were kept in a room maintained at $23 \pm 2^{\circ} \mathrm{C}$ and $50-65 \%$ humidity under a 12-h light/dark cycle (lights on at 7:00). Treatment and measurement were conducted according to the experimental schedules (Figure 1).

\section{A single-oral-administration of 5\% ELE and aroma treatment with $25 \mathrm{~g}$ ELE}

The rats were divided into three groups (Experiment 1 [Exp 1] consisting of control [Cont, $\mathrm{n}=5$ ], ELE 5\% [ $\mathrm{n}=4$ ], and ELE aroma $25 \mathrm{~g}$ [n = 4]; Experiment 2 [Exp 2] consisting of Cont [n =6], ELE $5 \%[\mathrm{n}=6]$, and ELE aroma $25 \mathrm{~g}[\mathrm{n}=5]$ ) based on the body weight before administration or treatment. On day 8 of Exp 1 (or day 14 of Exp 2), each rat of the Cont and ELE 5\% groups was orally administrated with distilled water and ELE dissolved in $1 \mathrm{~mL}$ distilled water through a probe once at 11:30. In ELE aroma group, we spread $25 \mathrm{~g}$ of ELE all over the container of an aluminum foil $8 \mathrm{~cm}$ in diameter in a small cage (W $150 \times$ D $150 \times$ H $150 \mathrm{~mm}$ ), which was connected to a metabolic cage through a tube. The metabolic cage was connected to a vacuum pump via another tube. The vacuum pump aspirated the air in the metabolic cage at a flow rate of $2.04 \mathrm{~L} / \mathrm{min}$, and successively aspirated the air of the small cage from the metabolic cage. The rat in a metabolic cage was continuously exposed to an aroma of ELE from a small cage as shown in Figure 2. On day 8 of Exp 1 (or day 14 of Exp 2), this aroma treatment was started at 11:30 and finished at 14:30.

\section{Ethics statement}

This study was conducted based on the "Guide for the Care and Use of Laboratory Animals" (NIH Publication No. 85-23, revised in 1996), and the experimental protocols were approved by the Ethics Committee on Animal Use of the Suzuka University of Medical Science (No. 121 of May 21, 2009).

\section{Experiment 1}

\section{Locomotor activity}

After conducting a 1-week preliminary breeding, a one-time 23hour measurement of rat's locomotor activity was performed for consecutive two days. The rats were moved to the metabolic cage (MK5000RQ, W $300 \times$ D $190 \times$ H 250 mm: MUROMACHI KIKAI CO., LTD., Tokyo, Japan). We accustomed a rat to a metabolism cage at the same time to investigate the 23 -h locomotor activity of the rat itself on day 7. After the measurement, the rats were temporarily moved to the home cage for $1 \mathrm{~h}$. After cleaning the metabolic cage, the same rats were returned to the metabolic cage and the locomotor activity was measured using the same method. On day 8 , rats were subjected to a single oral 5\% ELE administration just before the measurement of the locomotor activity or the aroma treatment with $25 \mathrm{~g}$ of ELE for $3 \mathrm{~h}$ after starting the measurement. The rats in the metabolic cage had free access to ND and water. Locomotor activity of rats was measured using an 
Miyazaki S (2017) Aroma of Eucommia leaf extract (ELE) causes reduced locomotor activity and increased NREM sleep, acting like the partially related factors of oral ELE's effects with locomotor-activity-dependent-increase in NREM- and REM-sleep
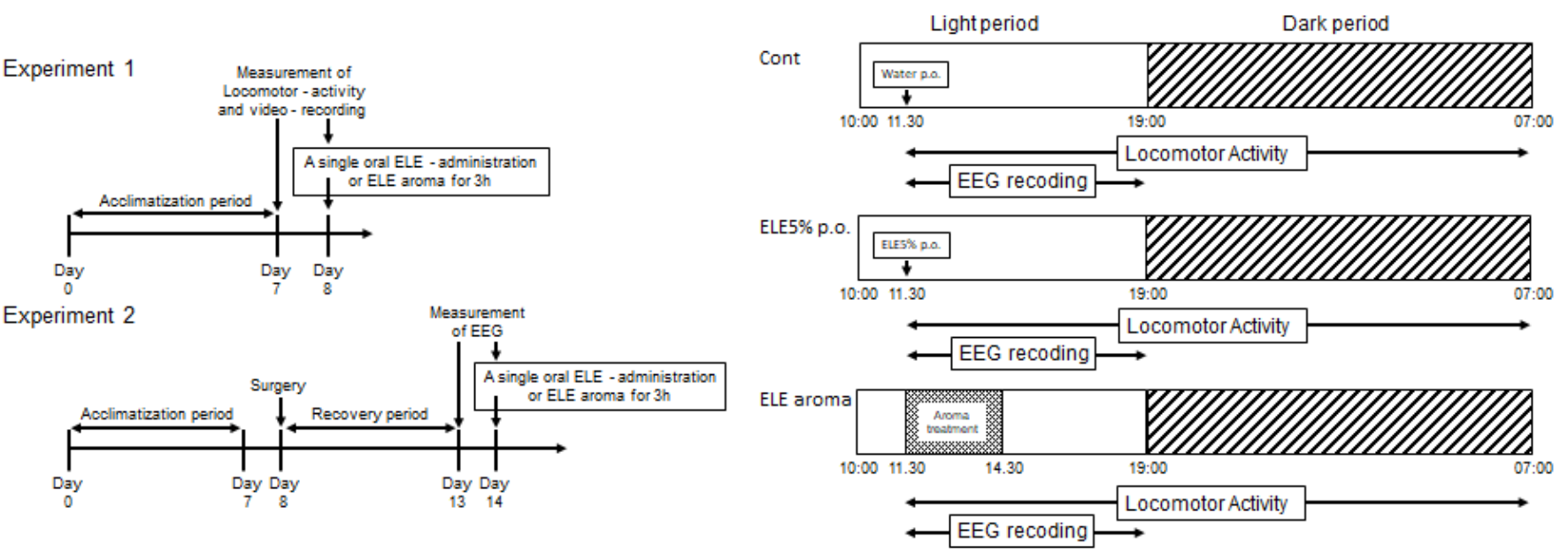

Figure 1. Experimental schedule for treatments with ELE and the measurement of the locomotor activity and the EEG. (A) Experimental schedule for treatments and measurements. (B) The time table in the Day 8

\section{(Aroma treatment)}
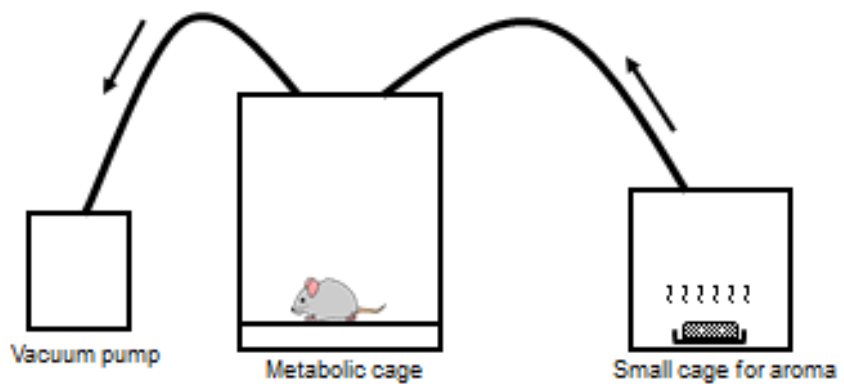

Figure 2. Experimental apparatus for the aroma treatment. The vacuum pump aspirated the air in the metabolic cage and successively aspirated the air of the small cage (which ELE $25 \mathrm{~g}$ in the container of aluminum foil of $8 \mathrm{~cm}$ in diameter was in) in a metabolic cage. The rat in a metabolic cage was continuously exposed for an aroma of ELE from a small cage.

infrared (IR) passive sensor system (Supermex; MUROMACHI KIKAI CO., LTD., Tokyo, Japan). The locomotor activity was monitored every $3 \mathrm{~min}$ for each IR beam break in the three planes ( $\mathrm{x}-, \mathrm{y}$-, and $\mathrm{z}$-axes). A video-recording analysis was simultaneously conducted to confirm the state (awakening and sleep) of rats. The locomotor activity analysis was carried out for $19.5 \mathrm{~h}$ (the time when human beings are not in the room: 11:30-07:00). From the analysis of the locomotor activity and the video-recoding, the rat's state was classified by the count per minute on the locomotor activity (simply 0 count/min as sleep, $1-199$ count $/ \mathrm{min}$ as rest, $\geqq 200 \mathrm{count} / \mathrm{min}$ as active). The time the rat spent sleeping, resting, and in activity was calculated in the entire period or in the light/dark periods.

\section{Experiment 2}

\section{Implantation surgery of EEG electrode}

Implanting of the EEG electrodes was performed by referring to the manual provided by emka Technologies (Falls Church, VA, US). Under anesthesia (somnopentyl; pentobarbital sodium, $50 \mathrm{mg} / \mathrm{kg}$, i.p.), the rats were fixed in a brain stereotaxic apparatus and their skull was exposed by making an incision on the skin from the head to the dorsal cervix along the midline. In rats, the electrodes were implanted in the left frontal cortex $(\mathrm{AP},-2.0 ; \mathrm{ML},-5.0)$ and the right parietal cortex (AP, $-2.0 ; \mathrm{ML},+5.0$ and $\mathrm{AP},-6.0 ; \mathrm{ML},+5.0)$ based on the rat brain atlas by Paxinos and Watson [38]. An electrode was inserted in the right parietal cortex $(\mathrm{AP},-6.0 ; \mathrm{ML},+5.0)$ as the reference. Two electromyography (EMG) wire electrodes were also inserted into both sides of the rat's dorsal neck muscle and were fixed. The socket (emka Technologies) that each electrode was connected to with a solder was fixed to the skull of the rats using a dental cement (SHOFU INC.). We attached a radiotransmitter ( $5.2 \mathrm{~g}$, emka Technologies) to the socket on the rat skull and confirmed reception states. After surgery, rats were returned to each cage. A recovery period of five days was established for the operated rats.

\section{EEG recording and analysis}

Since a single oral administration of ELE 5\% or aroma treatment with $25 \mathrm{~g}$ of ELE for $3 \mathrm{~h}$ induced sleep in the light phase in Exp 1, we conducted EEG recording to investigate which sleep stage influenced both oral and aroma ELE treatment routes. On the measurement day (Day 14), the rats were examined using the same methods as Exp 1. Using the rodentPACK (emka Technologies), data of EEG, EMG, and accelerometer (signals of posture and movement were composed of integrated movement along the $\mathrm{x}^{-}, \mathrm{y}^{-}$, and $\mathrm{z}$-axes) of the rats were simultaneously transmitted to the wireless receiver. The obtained data were used to analyze the sleep stages. The signal data (EEG, EMG, and accelerometer) without noise were acquired using the iox 2 software (emka Technologies). EEG data were analyzed using the ecgAUTO software to classify awake/sleep stages and differentiate between sleep stages. Local field potential (LFP) activity was amplified, filtered (sensitive, $100 \mathrm{~V} / \mu \mathrm{V}$; sampling rate, $200 \mathrm{~Hz}$ ), and digitized/stored at 3 $\mathrm{kHz}$. In classifying awake/sleep stages, the brain wave frequency band was measured using the delta band (as NREM sleep, 0.8-4 Hz), theta band (as REM sleep, 4-8 Hz), alpha band (as wakeful relaxation, 8-12 $\mathrm{Hz}$ ), and beta band (as awake, 12-22 Hz). A ten-second epoch scoring criterion was used. EEG recording started at 10:00 and finished at 09:00 on the next day. EEG analysis was performed for $7.5 \mathrm{~h}$ (light period, 11:30-19:00).

\section{Statistics analysis}

Values are expressed as means with standard errors derived from measurements of rats $(\operatorname{Exp} 1, \mathrm{n}=4-5$; $\operatorname{Exp} 2, \mathrm{n}=5-6)$. The statistical analysis was performed using SPSS statistics 22 (IBM, Tokyo, Japan). Homogeneity of variances was checked using the Levene's test. One-way analysis of variance (ANOVA) was used for inter- 
Miyazaki S (2017) Aroma of Eucommia leaf extract (ELE) causes reduced locomotor activity and increased NREM sleep, acting like the partially related factors of oral ELE's effects with locomotor-activity-dependent-increase in NREM- and REM-sleep

group comparisons. When the ANOVA results revealed significant differences, the Dunnett's $t$ or Dunnett's T3 post hoc test was used to identify significant differences between the Cont group and each ELEtreated group. The $p$-value of $<0.05$ was denoted as significant.

\section{Results}

\section{Experiment 1 (Locomotor activity)}

Effect of single oral administration of ELE 5\% and aroma treatment with ELE on locomotor activity: Rats orally administered with ELE 5\% exhibited a tendency toward decreased locomotor activity than that of the Cont group in the entire period (Figure 3). Rats treated with the aroma of ELE for $3 \mathrm{~h}$ exhibited a significant decrease in locomotor activity compared to that of the Cont group in the entire period. In the light period, a single oral administration of ELE 5\% or aroma treatment with ELE significantly decreased the locomotor activity compared to that of the Cont group ( $p<0.01$, Figure 3$)$. In the dark period, no significant difference was observed in the locomotor activity between the ELE 5\% and the Cont groups; however, the aroma group showed a significant decrease in locomotor activity $(p<0.01$, Figure 3).

Effect of single oral administration of ELE 5\% and aroma treatment with ELE on the time spent on sleeping, resting, and activity in the entire period or in the light/dark periods: The state (sleep, rest, and activity) of rats was classified based on their locomotor activity. Rats orally administered with ELE 5\% exhibited increasing tendency in the time spent for sleep ( 0 count $/ \mathrm{min}$ ) compared with the Cont group in the entire period (Figure 4A). Rats treated with aroma of ELE for $3 \mathrm{~h}$ exhibited significant increase in the time spent for sleep and rest (1-199 count/min) and a significant decrease in the time spent for activity ( $₫ 200$ count $/ \mathrm{min}$ ) compared with the Cont group (each $p<0.01$, Figure $4 \mathrm{~A}, 4 \mathrm{~B}$, and $4 \mathrm{C}$ ). In the light period, a single oral administration of ELE 5\% significantly increased the time spent for sleep and markedly decreased the time of rest compared with the Cont group $(p<0.05$ or $p<0.01$, Figure $4 \mathrm{~A}$ or $4 \mathrm{~B}$ ). Aroma treatment with ELE significantly increased the time spent for sleep and slightly decreased the time of rest compared with the Cont group $(p<0.05$ and $p<0.1$, respectively, Figure $4 \mathrm{~A}$ and $4 \mathrm{~B})$. In the dark period, no significant difference in the duration of all states was observed between the ELE 5\% group and the Cont group; however, the aroma group showed a significant increase in the duration of sleep and rest and a marked decrease in the duration

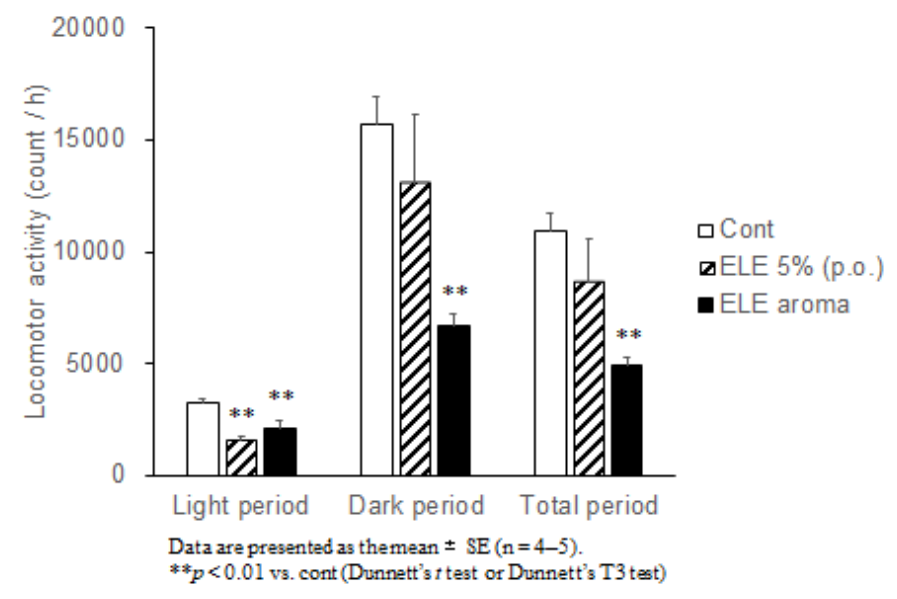

Figure 3. The effect of ELE 5\% (p.o.) and ELE aroma (25g) on the locomotor activity. Data are presented as the mean \pm SE $(\mathrm{n}=4-5) .{ }^{*} p<0.01 v s$. cont (Dunnett's t test or Dunnett's T3 test). of activity (each with $p$ of $<0.01$; Figure $4 \mathrm{~A}, 4 \mathrm{~B}$, and $4 \mathrm{C}$ ). The induction of sleeping time in the light period using a single oral administration of ELE 5\% was greatly associated with ELE's oral effect in the entire period (Figure 4A). The induction of the sleeping time was confirmed in rats treated with ELE aroma in both periods (Figure 4A).

\section{Experiment 2 (Classification of the rat's state based on EEG)}

Effects of single oral. administration of ELE 5\% and aroma treatment with ELE on the NREM sleep, REM sleep, wakeful relaxation, and awake states of rats in the light period: A single oral administration of ELE 5\% significantly increased the duration of NREM sleep and REM sleep ( $p<0.01$ or $p<0.05$, Figure 5A or 5B) and markedly decreased the duration of wakeful relaxation and wakefulness $(p<0.05$ or $p<0.01$, Figure 5C or 5D). The aroma treatment with ELE for $3 \mathrm{~h}$ significantly increased the duration of NREM sleep and markedly decreased the duration of wakefulness (each with $p$ of $<0.01$; Figure 5A and 5D). No significant difference in the duration of REM sleep and wakeful relaxation was observed between the ELE aroma and the Cont group (Figure 5B, 5C).

\section{Discussion}

This study showed that an oral administration of ELE and the aromatic treatment had the hypnotic effects, and those effects depend on the decrease in the locomotor activity using both treatments during an entire period, particularly in the light period. Then, the reduction of the locomotor activity in the light period using a single oral ELEadministration was based on the induction of the NREM and REM sleep. However, the reduction of the locomotor activity in the light period in an aromatic treatment with ELE was due to the induction of the NREM sleep. The result of this study was consistent with the sedative and hypnotic effects of $E$. ulmoides leaves reported in previous studies $[34,35]$. The mechanism of the induction of sleep by ELE is not well understood at this time. However, the oral administration of ELE seems to cause the activation of parasympathetic nervous system [31]. Parasympathetic nervous system induces the relaxation of the peripheral vascular system, which induces heat emission, resulting to sleep-onset time and sleepiness [39]. Thus, the relaxation of the peripheral vascular system might partially contribute to the hypnotic effect of the orally administered ELE. The mechanism of the hypnotic effect of the aromatic treatment of ELE is unknown. In a study investigating the hypnotic effect of aromatherapy, it was suggested that aromatic treatment may show the hypnotic effect via not only olfactory function but also sensory nerve endings located in the lungs and the lower airway [40]. The mechanism of the hypnotic effect using the aromatic treatment with ELE has to be addressed in future studies. In this study, a difference in the quality of the induced sleep was caused by the difference in the method of ELE treatment used. The aromatic treatment shows potential to have possibilities to act by a mechanism like the oral administration with ELE. Looking at a different perspective, the ELE aroma that resulted to reduction of locomotor activity and induction of NREM sleep may be partially related to the factors of oral ELE's effects with locomotor-activity-dependent increase in the NREM and REM sleep in the light period.

\section{Conclusion}

For treating insomnia, benzodiazepines that influence the sleep architecture and have the possibility of dependence, ramelteon that has a mild and hard to realize effects, or suvorexant that showed a narcolepsy-like symptom are clinically used. These medicines may be unsuitable for some people. However, the safety of ELE use has been 
Miyazaki S (2017) Aroma of Eucommia leaf extract (ELE) causes reduced locomotor activity and increased NREM sleep, acting like the partially related factors of oral ELE's effects with locomotor-activity-dependent-increase in NREM- and REM-sleep
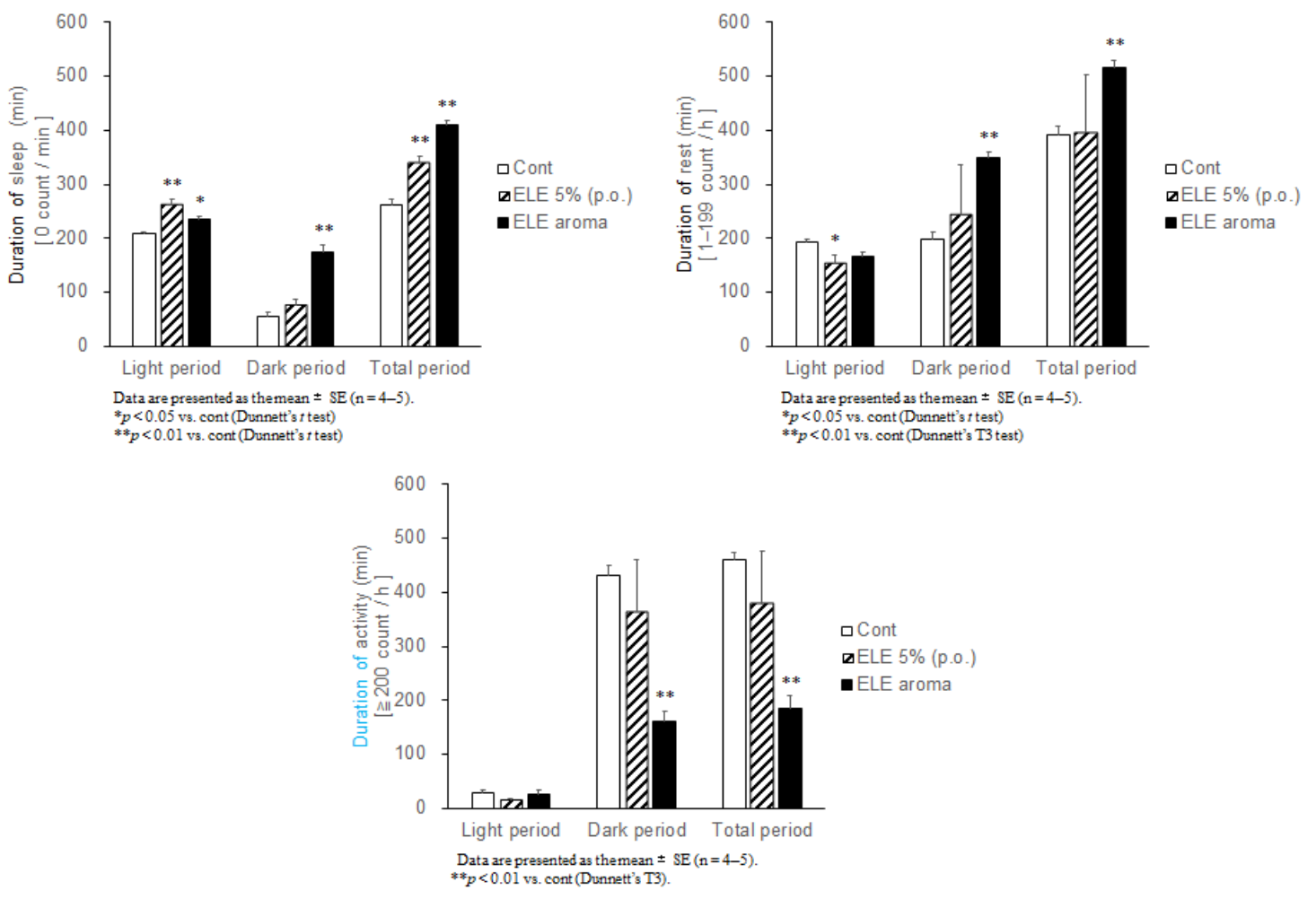

Figure 4. The effect of ELE 5\% (p.o.) and ELE aroma (25g) on the each state (sleep, rest and activity) of the rats. The rat's state was classified by the count per minute on the locomotor activity ( 0 count / $\mathrm{min}$ as sleep, $1-199$ count / $\mathrm{min}$ as rest, $\geqq 200$ count / $\mathrm{min}$ as active). The duration of each state in each period.

(A) The effect of ELE on the duration of sleep. (B) The effect of ELE on the duration of rest. (C) The effect of ELE on the duration of activity. Data are presented as the mean \pm SE ( $\mathrm{n}=4-5$ ). ${ }^{*} p<0.05,{ }^{*} p<0.01 v s$. Cont (Dunnett's $t$ test or Dunnett's T3).

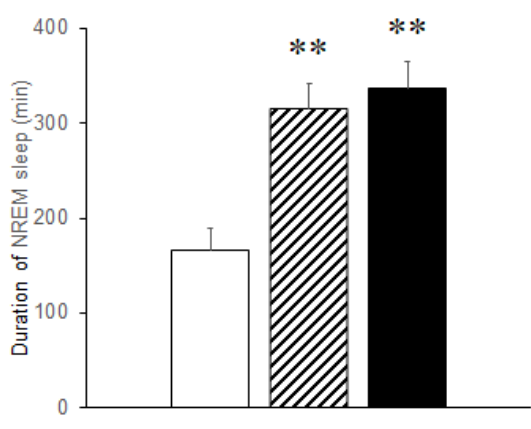

Data are presented as the mean $\pm \operatorname{SE}(n=5-6)$

$*^{* *} p<0.01$ vs. Cont (Dunnett's t test).

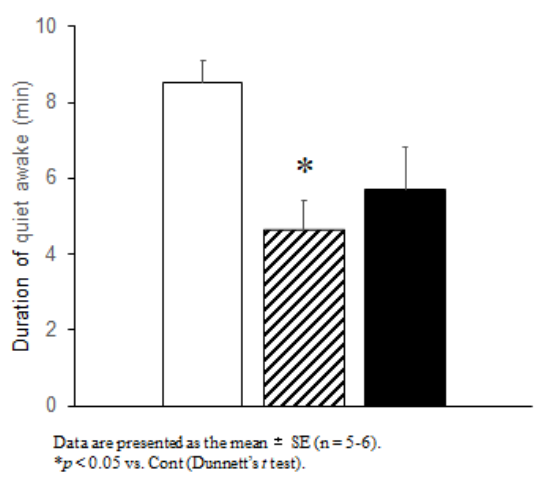

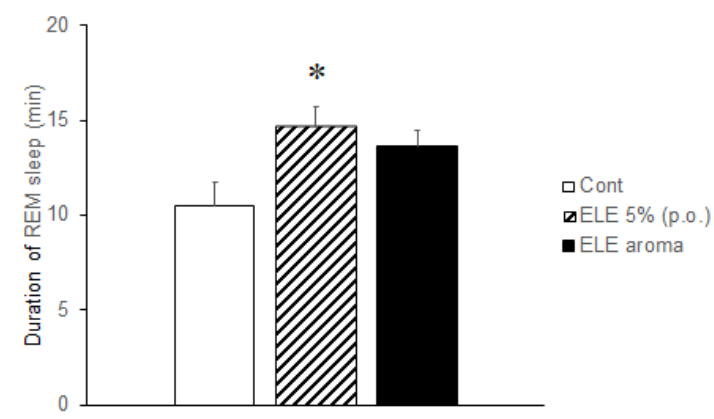

Data are presented as the mean $\pm \mathrm{SE}(\mathrm{n}=5-6)$

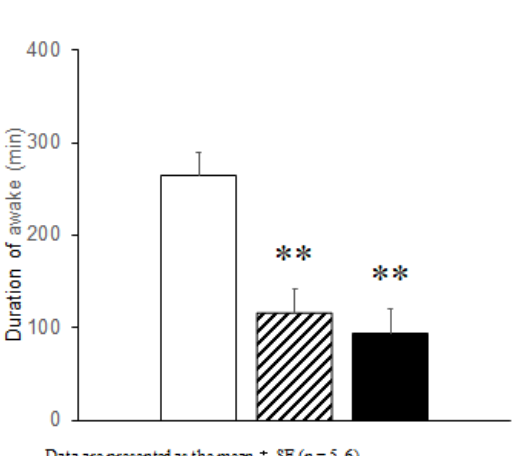

Data are presented as the mean $\pm \mathrm{SE}(\mathrm{n}=5-6)$.

Figure 5. The effect of ELE 5\% and ELE aroma (25g) on each sleep/awake stages. (A) The effect of ELE on the duration of NREM sleep ( $\delta$ wave: $0.3-4$ Hz). (B) The effect of ELE on the duration of REM sleep ( $\theta$ wave: $4-8 \mathrm{~Hz})$. (C) The effect of ELE on the duration of quiet awake ( $\alpha$ wave: $8-12 \mathrm{~Hz})$. (D) The effect of ELE on the duration of awake $(\beta$ wave: $12-22 \mathrm{~Hz})$. Data are presented as the mean $\pm \mathrm{SE} ; \mathrm{n}=5-6) .{ }^{*} p<0.05,{ }^{* *} p<0.01 v s$. Cont (Dunnett's $t$ test). 
Miyazaki S (2017) Aroma of Eucommia leaf extract (ELE) causes reduced locomotor activity and increased NREM sleep, acting like the partially related factors of oral ELE's effects with locomotor-activity-dependent-increase in NREM- and REM-sleep

previously reported [41]. Thus, instead of using these medicines, the two kinds of treatment methods using ELE may be the best choices in treating insomnia. Therefore, taking or smelling ELE improves daily lack of sleep and prevents the progression of insomnia.

\section{Acknowledgements}

The authors are grateful to M. Nakagawa for technical assistance and statistical analysis, S. Nakao for animal care. The study was performed with support from Kobayashi Pharmaceutical Co., Ltd. (Osaka, Japan). ELE was provided by Kobayashi Pharmaceutical Co., Ltd. The study sponsors had no role in the design and conduct of the study; the collection, management, analysis, and interpretation of the data; or the preparation, approval, and submission of the manuscript. All authors contributed to the writing of this manuscript and the interpretation of the results. All authors also reviewed the contents and approved the final version submitted for publication. H.O. and S.M. were the principal investigator of the study under the supervision of T.F.; N.H., N.K., T.H., S.H., Y.Y., and H.Y. contributed to the partial design of the study, data collection, and laboratory analysis. There are no financial or personal conflicts of interest in relation to the present study.

\section{References}

1. Baglioni C, Battagliese G, Feige B, Spiegelhalder K, Nissen C, et al. (2011) Insomnia as a predictor of depression: a meta-analytic evaluation of longitudinal epidemiological studies. J Affect Disord 135: 10-19. [Crossref]

2. Okajima I, Komada Y, Nomura T, Nakashima K, Inoue Y (2012) Insomnia as a risk for depression: a longitudinal epidemiologic study on a Japanese rural cohort. $J$ Clin Psychiatry 73: 377-383. [Crossref]

3. Troxel WM, Buysse DJ, Matthews KA, Kip KE, Strollo PJ, et al. (2010) Sleep symptoms predict the development of the metabolic syndrome. Sleep 33: 1633-1640. [Crossref]

4. Japanese ministry of health, labour and welfare (2015) National Health and Nutrition Survey in japan.

5. Schwartz MD, Kilduff TS (2015) The Neurobiology of Sleep and Wakefulness. Psychiatr Clin North Am 38: 615-644. [Crossref]

6. Monti JM (2013) The neurotransmitters of sleep and wake, a physiological reviews series. Sleep Med Rev 17: 313-315. [Crossref]

7. España RA, Scammell TE (2011) Sleep neurobiology from a clinical perspective. Sleep 34: 845-858. [Crossref]

8. Lillehei AS, Halcon LL (2014) A systematic review of the effect of inhaled essential oils on sleep. J Altern Complement Med 20: 441-451. [Crossref]

9. Perl O, Arzi A, Sela L, Secundo L, Holtzman Y, et al. (2016) Odors enhance slow-wave activity in non-rapid eye movement sleep. J Neurophysiol 115: 2294-2302. [Crossref]

10. Guizhou Province Institute for Drug Control and Guizhou Province Chinese Medicine Research Laboratories (1978) A hypertensive clinical study on Eucommia leaves. Chin Med Herbs Res 8: 59-63.

11. Guizhou Province Hypotensive Clinical Committee Institute for Drug Control and Chinese Medicine Research Laboratories (1978) A clinical study on replacement of Eucommia cortex with Eucommia leaves. New Med J 10: 30.

12. Liu D, Li CW (1980) Clinical evaluation of Eucommia cortex and leaves by 102 type of hypertension. Shanxi Chin Med 1: 27.

13. Shanxi Province Eucommia Clinical Review Committee (1995) Replacement of Eucommia Cortex with Eucommia Leaves for Hypertension Treatment. A Clinical Review with 621 Cases 19-54. Xian: Shanxi Eucommia Development Company.

14. Choi MS, Jung UJ, Kim HJ, Do GM, Jeon SM, et al. (2008) Du-zhong (Eucommia ulmoides Oliver) leaf extract mediates hypolipidemic action in hamsters fed a high-fat diet. Am J Chin Med 36: 81-93. [Crossref]

15. Horii Y, Tanida M, Shen J, Hirata T, Kawamura N, et al. (2010) Effects of Eucommia leaf extracts on autonomic nerves, body temperature, lipolysis, food intake, and body weight. Neurosci Lett 479: 181-186. [Crossref]
16. Park SA, Choi MS, Jung UJ, Kim MJ, Kim DJ, et al. (2006) Eucommia ulmoides Oliver leaf extract increases endogenous antioxidant activity in type 2 diabetic mice. $J \mathrm{Med}$ Food 9: 474-479. [Crossref]

17. Hirata T, Kobayashi T, Wada A, Ueda T, Fujikawa T, et al. (2011) Anti-obesity compounds in green leaves of Eucommia ulmoides. Bioorg Med Chem Lett 21: 17861791. [Crossref]

18. Jin X, Amitani K, Zamami Y, Takatori S, Hobara N, et al. (2010) Ameliorative effect of Eucommia ulmoides Oliv. leaves extract (ELE) on insulin resistance and abnormal perivascular innervation in fructose-drinking rats. J Ethnopharmacol 128: 672-678. [Crossref]

19. Fujikawa T, Hirata T, Wada A, Kawamura N, Yamaguchi Y, et al. (2010) Chronic administration of Eucommia leaf stimulates metabolic function of rats across several organs. Br J Nutr 104: 1868-1877. [Crossref]

20. Kobayashi Y, Hiroi T, Araki M, Hirokawa T, Miyazawa M, et al. (2012) Facilitative effects of Eucommia ulmoides on fatty acid oxidation in hypertriglyceridaemic rats. $J$ Sci Food Agric 92: 358-365. [Crossref]

21. Zhang W, Fujikawa T, Mizuno K, Ishida T, Ooi K, et al. (2012) A Eucommia leaf extract (ELE) prevents OVX-induced osteoporosis and obesity in rats. Am J Chin Med 40: 735-752. [Crossref]

22. Lin J, Fan YJ, Mehl C, Zhu JJ, Chen H, et al. (2011) Eucommia ulmoides Oliv antagonizes $\mathrm{H} 2 \mathrm{O} 2$-induced rat osteoblastic MC3T3-E1 apoptosis by inhibiting expressions of caspases 3, 6, 7, and 9. J Zhejiang Univ Sci B 12: 47-54. [Crossref]

23. Zhang R, Liu ZG, Li C, Hu SJ, Liu L, et al. (2009) Du-Zhong (Eucommia ulmoides Oliv.) cortex extract prevent OVX-induced osteoporosis in rats. Bone 45: 553-559. [Crossref]

24. Li Y, Wang MJ, Li S, Zhang YM, Zhao Y, et al. (2011) Effect of total glycosides from Eucommia ulmoides seed on bone microarchitecture in rats. Phytother Res 25: 18951897. [Crossref]

25. Ong VY, Tan BK (2007) Novel phytoandrogens and lipidic augmenters from Eucommia ulmoides. BMC Complement Altern Med 7: 3. [Crossref]

26. Ha H, Ho J, Shin S, Kim H, Koo S, et al. (2003) Effects of Eucommiae Cortex on osteoblast-like cell proliferation and osteoclast inhibition. Arch Pharm Res 26: 929936. [Crossref]

27. Jiang L, Wang Z, Zhu HW, Di HY, Li H, et al. (2011) Beneficial effect of Eucommia polysaccharides on systemic lupus erythematosus-like syndrome induced by Campylobacter jejuni in BALB/c mice. Inflammation 34: 402-411. [Crossref]

28. Kwon SH, Lee HK, Kim JA, Hong SI, Kim SY, et al. (2011) Neuroprotective effects of Eucommia ulmoides Oliv. Bark on amyloid beta(25-35)-induced learning and memory impairments in mice. Neurosci Lett 487: 123-127. [Crossref]

29. Zhou Y, Liang M, Li W, Li K, Li P, et al. (2009) Protective effects of Eucommia ulmoides Oliv. bark and leaf on amyloid $\beta$-induced cytotoxicity. Environ Toxicol Pharmacol 28: 342-349.

30. Okada N, Shirata K, Niwano M, Koshino H, Uramoto M (1994) Immunosuppressive activity of a monoterpene from Eucommia ulmoides. Phytochemistry 37: 281-282.

31. Yamaguchi Y, Kawamura N, Tsuboi T, Yamaguchi Y, Hirata T, et al. (2007) Effect of the Eucommia ulmoides Leaf Extract on Blood Pressure. Int Symp Eucommia ulmoides 1: $55-62$

32. Tagawa C, Nakazawa Y, Tagashira E, Ueda T, Yamaguchi Y, et al. (2005) Effect of Eucommia Leaf (Eucommia ulmoides Oliver ; Du-Zhong yge) Extract on Blood Pressure (2) [in Japanese]. Nat Med 59: 117-120.

33. Yan JK, Ding LQ, Shi XL, Donkor PO, Chen LX, et al. (2017) Megastigmane glycosides from leaves of Eucommia ulmoides Oliver with ACE inhibitory activity. Fitoterapia 116: 121-125. [Crossref]

34. Li X, Zhu W, Yang L, Fei D, Fan J, et al. (2013) Evaluation of the sedative and hypnotic effects of Eucommiol in Eucommia. Nat Prod Res 27: 1657-1659. [Crossref]

35. Li X, Tang Z, Fei D, Liu Y, Zhang M, et al. (2016) Evaluation of the sedative and hypnotic effects of astragalin isolated from Eucommia ulmoides leaves in mice. Nat Prod Res 26: 1-5. [Crossref]

36. Fujikawa T, Hirata T, Hosoo S, Nakajima K, Wada A, et al. (2012) Asperuloside stimulates metabolic function in rats across several organs under high-fat diet conditions, acting like the major ingredient of Eucommia leaves with anti-obesity activity. J Nutr Sci 1: e10. [Crossref]

37. Oikawa H, Nakamich S, Nishide H, Kawaguchi N, Zhang W, et al. (2016) Engagemen of the aroma in feeding suppression behavior of Eucommia leaf[in Japanese]. J Aroma Sci Technol Safety 17: 74-79. 
Miyazaki S (2017) Aroma of Eucommia leaf extract (ELE) causes reduced locomotor activity and increased NREM sleep, acting like the partially related factors of oral ELE's effects with locomotor-activity-dependent-increase in NREM- and REM-sleep

38. Paxinos G, Watoson C (1986) The rat brain in stereotaxic coordinates. $2^{\text {nd }}$ ed. San Diego: Academic Press.

39. Kräuchi K, Cajochen C, Werth E, Wirz-Justice A (1999) Warm feet promote the rapid onset of sleep. Nature 401: 36-37. [Crossref]
40. Kagawa D, Jokura H, Ochiai R, Tokimitsu I, Tsubone H (2003) The sedative effects and mechanism of action of cedrol inhalation with behavioral pharmacological evaluation. Planta Med 69: 637-641. [Crossref]

41. Kawasaki T, Uezono K, Nakazawa Y (2000) Antihypertensive mechanism of food for specified health use: "Eucommia leaf glycoside" and its clinical application. J. Health Sci 22: 29-36.

Copyright: (2017 Miyazaki S. This is an open-access article distributed under the terms of the Creative Commons Attribution License, which permits unrestricted use, distribution, and reproduction in any medium, provided the original author and source are credited. 